Pacific Journal of Mathematics

ON UNIVERSALLY COMPLETE RIESZ SPACES 


\title{
ON UNIVERSALLY COMPLETE RIESZ SPACES
}

\author{
C. D. Aliprantis and O. Burkinshaw
}

In Math. Proc. Cambridge Philos. Soc. (1975), D. H. Fremlin studied the structure of the locally solid topologies on inextensible Riesz spaces. He subsequently conjectured that his results should hold true for $\sigma$-universally complete Riesz spaces.

In this paper we prove that indeed Fremlin's results can be generalized to $\sigma$-universally complete Riesz spaces and at the same time establish a number of new properties. Examples of Archimedean universally complete Riesz spaces which are not Dedekind complete are also given.

1. Preliminaries. For notation and terminology concerning Riesz spaces not explained below we refer the reader to [10].

A Riesz space $L$ is said to be universally complete if the supremum of every disjoint system of $L^{+}$exists in $L$ [10, Definition 47.3, p. 323]; see also [12, p. 140]. Similarly a Riesz space $L$ is said to be $\sigma$-universally complete if the supremum of every disjoint sequence of $L^{+}$exists in $L$. An inextensible Riesz space is a Dedekind complete and universally complete Riesz space [7, Definition (a), p. 72]. In the terminology of [12] an inextensible Riesz space is called an extended Dedekind complete Riesz space; see [12, pp. 140-144].

Every band in a universally complete Riesz space is in its own right a universally complete Riesz space. Archimedean universally complete Riesz spaces possess weak order units (the supremum of any maximal disjoint system of positive elements must be a weak order unit) and their bands are principal bands, since every band possesses a weak order unit. Remarkably, Archimedean $\sigma$-universally complete Riesz spaces satisfy the principal projection property (and hence Archimedean universally complete Riesz spaces satisfy the projection property) [11, Theorem 8, p. 32] and [5, Theorem 1, p. 320].

A Riesz subspace $L$ of a Riesz space $M$ is said to be order dense in $M$ if $u=\sup \{v \in L: \theta \leqq v \leqq u\}$ holds in $M$ for each $u \in M^{+}$(if $M$ is Archimedean this is equivalent to the property: For each $\theta<u \in M$, there exists $v \in L$ with $\theta<v \leqq u) . \quad L$ is said to be full in $M$ if for every $u \in M$ there exists $v \in L$ with $|u| \leqq v$.

For the the proof of the next theorem follow the proof of $[5$, Theorem 2, p. 321] or see [7].

THEOREM 1.1. Let $L$ be an order dense Riesz subspace of an Archimedean Riesz space $M$. Then we have: 
(i) If $L$ is universally complete, then $L$ is full in $M$.

(ii) If $L$ is inextensible, then $L=M$.

Every Archimedean Riesz space can be embedded as an order dense Riesz subspace in a uniquely determined (up to a Riesz isomorphism) inextensible Riesz space $L^{u}$, called the universal completion of $L$ [10, Theorem 50.8, p. 340].

A nonempty subset $A$ of positive elements of a Riesz space $L$ is said to be a dominable subset of $L^{+}$if for every $\theta<u \in L$ there exists a positive integer $k$ and $\theta<v \in L$ such that $\theta<v \leqq(k u-\alpha)^{+}$ holds in $L$ for all $a \in A$ [7, Definition (c), p. 72]. Observe that if $L$ is order dense in $M$, then a nonempty subset $A$ of $L^{+}$is a dominable subset of $L^{+}$if and only if $A$ is a dominable subset of $M^{+}$. This observation applied to the universal completion $L^{u}$, combined with Theorem 1.1 and [7, Theorem 1.4, p. 74] yields the following result.

Theorem 1.2. A nonempty subset $A$ of $L^{+}$of an Archimedean universally complete Riesz space $L$ is dominable if, and only if, $A$ is order bounded in $L$.

We recall that for a topologeal space $X$ the set $C^{\infty}(X)$ denotes the set of all extended real valued continuous functions $f$ on $X$ for which the set $\{x \in X:|f(x)|<\infty\}$ is dense in $X$. If $X$ is extremally disconnected (i.e., if the closure of every open set of $X$ is also open) then $C^{\infty}(X)$ forms an inextensible Riesz space under the ordinary operations [10, Theorem 47.4, p. 323]. By a step function $s$ on a topological space $X$ we mean a function $s \in C^{\infty}(X)$ for which there exists a collection $\left\{V_{i}: i \in I\right\}$ of mutually disjoint closed-open subsets of $X$ such that $s$ is constant on each $V_{i}$ and with the open set $\mathscr{O}=\mathbf{U}\left\{V_{i}: i \in I\right\}$ dense in $X$. The collection of all step functions on $X$ will be denoted by $S^{\infty}(X)$.

Regarding $S^{\circ}(X)$ we have the following theorem whose straightforward proof is left to the reader.

THEOREM 1.3. Let $X$ be an extremally disconnected topological space. Then $S^{\infty}(X)$ is an Archimedean universally complete Riesz space.

From the representation theorems of the Archimedean Riesz spaces and [5, Theorem 1, p. 320] the following result should be clear now.

THEOREM 1.4. For every Archimedean universally complete Riesz space $L$ there exists a compact, Hausdorff extremally disconnected topological space $X$ satisfying the following Riesz subspace inclusions: 


$$
S^{\infty}(X) \subseteq L \subseteq C^{\infty}(X)
$$

We continue with examples of universally complete Riesz spaces.

EXAMPle 1.5. (i) (An Archimedean universally complete Riesz space which is not Dedekind complete.) By [8, Theorem 3.2, p. 486] there exists a compact, Hausdorff extremally disconnected topological space $X$ and an onto continuous function $\varphi: X \rightarrow[0,1]$, such that $\varphi$ does not carry any proper closed subset of $X$ onto $[0,1]$. Note that $\varphi \in C(X) \subseteq C^{\infty}(X)$. We shall show that $\varphi \notin S^{\infty}(X)$. Indeed, if $\varphi \in$ $S^{\infty}(X)$ then $\varphi$ must be constant on some nonempty proper closed-open subset $V$ of $X$. But then $\varphi$ maps the closed proper subset $X \sim V$ onto $[0,1]$, in contradiction with the properties of $\varphi^{1}$. Now if $S^{\infty}(X)$ is Dedekind complete then $S^{\infty}(X)=C^{\infty}(X)$ by Theorem 1.1, in contradiction to $\varphi \notin S^{\infty}(X)$. Thus $S^{\infty}(X)$ is the desired example.

(ii) (An Archimedean $\sigma$-universally complete Riesz space which is neither $\sigma$-Dedekind complete nor universally complete.) If $K$ is the Riesz space of all real valued Lebesgue measurable function defined on $[0,1]$ (with the pointwise ordering) and $S^{\infty}(X)$ is as determined in (i) above, then the Riesz space $L=K \times S^{\infty}(X)$ has the stated properties.

A Riesz space $L$ is said to satisfy the countable sup property if for every subset $S$ of $L$ whose supremum exists in $L$ there exists an at most countable subset of $S$ having the same supremum as $S$ in $L$.

A net $\left\{u_{\alpha}\right\}$ of a Riesz space $L$ order converges to $u$ in $L$, denoted by $u_{\alpha} \stackrel{(o)}{\rightarrow} u$, if there exists a net $\left\{v_{\alpha}\right\}$ of $L$ (with the same directed indexed set) such that $\left|u_{\alpha}-u\right| \leqq v_{\alpha} \downarrow \theta$ holds in $L$. A subset $S$ of $L$ is said to be a solid set if $|u| \leqq|v|$ in $L$ and $v \in S$ implies $u \in S$ and $S$ is said to be order closed if $u_{\alpha} \stackrel{(o)}{\rightarrow} u$ in $L$ and $\left\{u_{\alpha}\right\} \subseteq S$ implies $u \in S$. If $S$ is both solid and order closed then $S$ is said to satisfy the Fatou property.

A locally solid topology $\tau$ on a Riesz space $L$ is a linear topology $\tau$ having a basis for zero consisting of solid sets. A locally solid Riesz space $(L, \tau)$ is a Riesz space $L$ equipped with a locally solid topology $\tau$. For our discussion we shall need the following properties of locally solid Riesz spaces $(L, \tau)$ connecting order and topological continuity.

$\sigma$-Lebesgue property: $u_{n} \downarrow \theta$ in $L$ implies $u_{n} \stackrel{\tau}{\rightarrow} \theta$.

Lebesgue property: $u_{\alpha} \downarrow \theta$ in $L$ implies $u_{\alpha} \stackrel{\tau}{\rightarrow} \theta$.

1 This proof was suggested by Professor J. Quinn. 
Pre-Lebesgue property: $\theta \leqq u_{n} \uparrow \leqq u$ in $L$ implies $\left\{u_{n}\right\}$ is a $\tau$-Cauchy sequence.

Fatou property: $\tau$ has a basis for zero consisting of sets with the Fatou property.

The terminology of the above properties is due to D. H. Fremlin [6]. In the terminology of Luxemburg and Zaanen the $\sigma$-Lebesgue, Lebesgue and pre-Lebesgue properties are referred to as the $(A, \mathrm{i})$, $(A$, ii) and $(A$, iii) properties respectively [9, p. 501]. It should be noted that the pre-Lebesgue property is equivalent to the condition: $\theta \leqq u_{\alpha} \uparrow \leqq u$ in $L$ implies that $\left\{u_{\alpha}\right\}$ is a $\tau$-Cauchy net; see [1, Theorem 2.1(2), p. 443] and [9, p. 502].

2. Some results concerning Fatou and Lebesgue topologies. Let $(L, \tau)$ be a locally solid Riesz space. We shall call a sequence $\left\{V_{n}\right\}$ of $\tau$-neighborhoods of zero normal if $V_{n+1}+V_{n+1} \subseteq V_{n}$ holds for every $n=1,2, \cdots$. We define the null ideal $N$ of a normal sequence $\left\{V_{n}\right\}$ of solid $\tau$-neighborhoods of zero by

$$
N=\bigcap\left\{V_{n}: n=1,2, \cdots\right\} .
$$

It follows that $N$ is an ideal of $L$, and if the normal sequence $\left\{V_{n}\right\}$ consists of Fatou $\tau$-neighborhoods of zero, then $N$ is also a band of $L$.

The carrier of a locally solid topology $\tau$ on a Riesz space is defined by

$$
\begin{aligned}
C_{\tau}=\bigcup\left\{N^{d}: N=\bigcap_{n=1}^{\infty} V_{n} ;\left\{V_{n}\right\}\right. \text { is a normal } \\
\text { sequence of solid neighborhoods }\} .
\end{aligned}
$$

Observe that $C_{\tau}$ is an ideal of $L$. This ideal seems to have been first introduced by D. H. Fremlin; see [6, 23L Proposition, p. 50]. Some of the basic properties of the carrier are included in the next lemma.

Lemma 2.1. Let $(L, \tau)$ be a Hausdorff locally solid Riesz space. Then we have:

(i) The carrier $C_{\tau}$ of $\tau$ is a $\sigma$-ideal of $L$.

(ii) If $\tau$ satisfies the Fatou property, then $C_{\tau}$ is an order dense $\sigma$-ideal (and hence in this case, if $L$ has the countable sup property, $\left.C_{\tau}=L\right)$.

(iii) If $\tau$ satisfies the Lebesgue property, then $C_{\tau}$ has the countable sup property.

Proof. (i) Assume that $\theta \leqq u_{n} \uparrow u$ holds in $L$ with $\left\{u_{n}\right\} \leqq C_{\tau}$. For each $i$ pick a normal sequence $\left\{V_{n}^{i}: n=1,2, \cdots\right\}$ of solid $\tau$-neighborhoods of zero such that $u_{i} \in N_{i}^{d} ; N_{i}=\bigcap\left\{V_{n}^{i}: n=1,2, \cdots\right\}, i=$ 
$1,2, \cdots$. Set $W_{n}=\bigcap\left\{V_{n}^{i}: i=1, \cdots, n\right\}$ for $n=1,2, \cdots$ and note that $\left\{W_{n}\right\}$ is a normal sequence of solid $\tau$-neighborhoods of zero satisfying

$$
N=\bigcap\left\{W_{n}: n=1,2, \cdots\right\} \subseteq \bigcap\left\{V_{n}^{i}: n=1,2, \cdots\right\}=N_{i}
$$

for $i=1,2, \cdots$. Thus $N_{\imath}^{d} \subseteq N^{d}$ for $i=1,2, \cdots$ and so $\left\{u_{n}\right\} \subseteq N^{d}$. But then $u \in N^{d} \cong C_{\tau}$ and thus $C_{\tau}$ is a $\sigma$-ideal of $L$.

(ii) Let $\theta<u \in L$. Pick a Fatou $\tau$-neighborhood $V$ of zero of $L$ with $u \notin V$. Next choose a normal sequence $\left\{V_{n}\right\}$ of Fatou $\tau$-neighborhoods of zero with $V_{1}=V$. Now since $L$ is Archimedean and $N=\bigcap\left\{V_{n}: n=1,2, \cdots\right\}$ is a band, $N=N^{d d}$ and so (since $u \notin N$ ) there oxists $\theta \leqq v \in N^{d} \leqq C_{\text {s }}$ such that $w=u \wedge v>\theta$. But then $w \in C_{\tau}$ satisfies $\theta<w \leqq u$, so that $C_{\tau}$ is order dense in $L$ (this argument, due to the referee, replaces our somewhat involved proof). The parenthetical part follows now from part (i).

(iii) Assume $u_{\alpha} \downarrow \theta$ in $C_{\tau}$. We can assume $\theta \leqq u_{\alpha} \leqq u$ holds in $C_{\tau}$ for each $\alpha$ and some $u \in C_{\tau}$. Pick a normal sequence $\left\{V_{n}\right\}$ of Fatou $\tau$-neighborhoods of zero with $u \in N^{d}$. So, $\left\{u_{\alpha}\right\} \subseteq N^{d}$. Since $\tau$ is a Lebesgue topology $u_{\alpha} \stackrel{\tau}{\rightarrow} \theta$. Now for each $n$ pick $u_{\alpha_{n}} \in V_{n}$. We can assume $u_{\alpha_{n}} \downarrow$ in $C_{\tau}$. It follows now easily that $u_{\alpha_{n}} \downarrow \theta$ holds in $C_{\tau}$ and hence $C_{\text {. }}$ has the countable sup property.

The next result is a corollary of the previous lemma and will be used later.

THEOREM 2.2. If a Riesz space $L$ with the countable sup property and a weak order unit admits a Hausdorff Fatou (resp. Lebesgue) topology $\tau$, then it admits also a metrizable Fatou (resp. Lebesgue) topology $\tau^{\prime}$ coarser than $\tau$ (i.e., $\tau^{\prime} \leqq \tau$ ).

Proof. By Lemma 2.1(ii) we have $C_{\tau}=L$. Now, since $L$ has a weak order unit there exists a normal sequence $\left\{V_{n}\right\}$ of Fatou $\tau$ neighborhoods of zero such that $L=N^{d}$, where $N=\bigcap\left\{V_{n}: n=\right.$ $1,2, \cdots\}$. But then $\left\{V_{n}\right\}$ defines a metrizable Fatou topology $\tau^{\prime}$ on $L$ (which must be necessarily Lebesgue, if $\tau$ is Lebesgue) satisfying $\tau^{\prime} \cong \tau$.

I. Amemiya [4, Theorem (B, 4), p. 21] obtained one of the deepest property of the Fatou topologies by using a Baire type categorical argument. We are now in the position to generalize and give a straightforward proof of this result by using simple Riesz space arguments. 
Hausdorff Fatou topology $\tau_{1}$ and a Lebesgue topology $\tau_{2}$. Then the topology induced by $\tau_{1}$ on any order bounded subset of $L$ is finer than the topology induced by $\tau_{2}$ on the same set.

Proof. According to [3, Theorem 2.1, p. 26] we can assume that $L$ is Dedekind complete. Now let $\left\{u_{\alpha}\right\} \subseteq[\theta, u]$ satisfying $u_{\alpha} \stackrel{\tau_{1}}{\rightarrow} \theta$. We have to show that $u_{\alpha} \stackrel{\tau_{2}}{\rightarrow} \theta$. Assume that this is not the case. Then there exist two solid $\tau_{2}$-neighborhoods of zero $V$ and $U$ with $V+V \subseteq U$ and with the property that for any $\alpha$ there exists $\beta \geqq \alpha$ with $u_{\beta} \notin U$.

Now since $C_{\tau_{1}}$ is order dense in $L$ and $\tau_{2}$ is a Lebesgue topology there exist $w \in C_{\tau_{1}}$ with $\theta \leqq w \leqq u$ and $u-w \in V$. Pick a normal sequence $\left\{V_{n}\right\}$ of Fatou $\tau_{1}$-neighborhoods of zero with $w \in N^{d}$. Since $w \wedge u_{\alpha} \stackrel{\tau_{1}}{\rightarrow} \theta$ there exists a sequence of indices $\left\{\alpha_{n}\right\}$ with $u_{\alpha_{n}} \notin U$ and $w \wedge u_{\alpha_{n}} \in V_{n+1}$ for all $n$. It follows now easily that $w_{1}=$ $\lim \sup w \wedge u_{\alpha_{n}} \in V_{n}$ holds for all $n$. Thus $w_{1} \in N \cap N^{d}=\{\theta\}$ holds and so $w \wedge u_{\alpha_{n}} \stackrel{(o)}{\rightarrow} \theta$ holds in $L$. But then $w \wedge u_{\alpha_{n}} \stackrel{\tau_{2}}{\rightarrow} \theta$ since $\tau_{2}$ is a Lebesgue topology and so

$$
\begin{aligned}
\theta & \leqq u_{\alpha_{n}}=\left(u_{\alpha_{n}}-w\right)^{+}+w \wedge u_{\alpha_{n}} \\
& \leqq(u-w)+w \wedge u_{\alpha_{n}} \in V+V \leqq U
\end{aligned}
$$

for $n$ sufficiently large, in cotradiction with the choice of $\left\{u_{\alpha_{n}}\right\}$. This shows that $u_{\alpha} \stackrel{\tau_{2}}{\rightarrow} \theta$ and the proof is finished.

The next result is an obvious corollary of the previous theorem and it also generalizes a result of I. Amemiya [4, Theorem $(B, 5)$, p. 22].

THEOREM 2.4. All the Hausdorff Lebesgue topologies on a Riesz space $L$ induce the same topology on the order bounded subsets of $L$.

It is possible for a Riesz space to admit more than one Hausdorff Lebesgue topology. This possibility as well as Theorem 2.3 are illustrated in the next examples.

Example 2.5. (i) Let $L$ be the super Dedekind complete Riesz space consisting of the equivalence classes of the bounded (almost everywhere) Lebesgue measurable functions on $[0,1]$. Let $\tau_{1}$ and $\tau_{2}$ be the Hausdorff Lebesgue topologies generated respectively by the Riesz norms 


$$
\rho_{1}(u)=\int_{0}^{1}|u(x)| d x \text { and } \rho_{2}(u)=\left(\int_{0}^{1}|u(x)|^{2} d x\right)^{1 / 2} \text { for } u \in L .
$$

Note that $\tau_{1} \neq \tau_{2}$. Actually the Hausdorff Lebesgue topologies $\left\{\tau_{p}: 1 \leqq p<\infty\right\}$ generated by the Riesz norms $\rho_{p}(u)=\left(\int_{0}^{1}|u(x)|^{p} d x\right)^{1 / p}$ are all dinstinct for $1 \leqq p<\infty$.

(ii) Let $X$ be an infinite set and let $L=l^{\infty}(X)$. Let $\tau_{1}$ be the Hausdorff Fatou topology (but not Lebesgue) generated by the sup norm and $\tau_{2}$ the Hausdorff Lebesgue topology of the pointwise convergence. Then $\tau_{2} \subseteq \tau_{1}$, but $\tau_{1} \neq \tau_{2}$. Compare this with Theorem 2.3.

The next result tells us about the relation between the countable sup property and Hausdorff Lebesgue topologies.

THEOREM 2.6. Let $(L, \tau)$ be a Hausdorff locally solid Riesz space with the Lebesgue property. Then the following statements are equivalent:

(i) L has the countable sup property.

(ii) $C_{\tau}=L$ (where $C_{\tau}$ is the carrier of $\tau$ ).

(iii) $\tau$ induces a metrizable topology on each order interval of $L$.

Proof. (i) $\Rightarrow$ (ii). It follows immediately from Lemma 2.1 (ii).

(ii) $\Rightarrow$ (iii). Let $u \in L^{+}$. Then $[\theta, u] \subseteq N^{d}$ must hold for some normal sequence $\left\{V_{n}\right\}$ of Fatou $\tau$-neighborhoods of zero, where $N=$ $\bigcap\left\{V_{n}: n=1,2, \cdots\right\}$. Now $\tau$ restricted to $N^{d}$ satisfies the Lebesgue property and $\left\{V_{n}\right\}$ defines a metrizable topology on $N^{d}$. But then according to Theorem 2.4 both topologies agree on $[\theta, u]$ and so $\tau$ induces a metrizable topology on $[\theta, u]$.

(iii) $\Rightarrow$ (i). Assume $u_{\alpha} \downarrow \theta$ in $L$. Without loss of generality we can suppose that $\theta \leqq u_{\alpha} \leqq u$ holds in $L$ for each $\alpha$. Let $\left\{V_{n}\right\}$ be a normal sequence of Fatou $\tau$-neighborhoods of zero such that $\left\{V_{n} \cap[\theta, u]\right\}$ is a basis of zero for the topology induced on $[\theta, u]$ by $\tau$. For each $n$ pick an index $\alpha_{n}$ with $u_{\alpha_{n}} \in V_{n} \cap[\theta, u]$ (this is possible since the topology $\tau$ is Lebesgue). We can assume $u_{\alpha_{n}} \downarrow$ in $L$. If $\theta \leqq v \leqq u_{\alpha_{n}}$ holds for all $n$ in $L$, then $v \in V_{n} \cap[\theta, u]$ also holds for all $n$ and hence $u_{\alpha_{n}} \downarrow \theta$ in $L$. This shows that $L$ has the countable sup property.

3. Locally solid topologies on universally complete Riesz spaces. In this section we study the locally solid topologies that a $\sigma$-universally complete Riesz space can carry and in the process generalize the results obtained by D. H. Fremlin in [7, §2, pp. 80-84]. We start with the following theorem. 
THeOREM 3.1. Let $L$ be an Archimedean $\sigma$-universally complete Riesz. Then we have:

(i ) Every disjoint sequence of $L^{+}$converges to zero with respect to any locally solid topology on $L$. In particular every locally solid topology on $L$ satisfies the pre-Lebesgue property.

(ii) Every locally solid topology on $L$ satisfies the $\sigma$-Lebesgue property.

Proof. (i) Part (i) follows immediately from [6, 24H Proposition, p. 56].

(ii) Let $\tau$ be a locally solid topology on $L$ and let $u_{n} \downarrow \theta$ in $L$. Let $V$ be a solid $\tau$-neighborhood of zero. Choose another $\tau$-neighborhood of zero $U$ with $U+U \subseteq V$. Pick $\delta>0$ such that $\delta u_{1} \in U$. By [5, Theorem 1] $L$ satisfies the principal projection property. Let $P_{n}$ denote the projection on the band generated by $\left(u_{n}-\delta u_{1}\right)^{+}$. Put $v_{n}=P_{n}\left(u_{1}\right), w_{n}=u_{1}-v_{n}$ for all $n$ and observe that $w_{n} \wedge\left(u_{n}-\delta u_{1}\right)^{+}=\theta$. Since $\left(u_{n}-\delta u_{1}\right)^{+}=P_{n}\left(u_{n}-\delta u_{1}\right)=P_{n}\left(u_{n}\right)-\delta v_{n}$ we get $\theta \leqq \delta v_{n} \leqq u_{n}$ and so $v_{n} \downarrow \theta$. Note next that $\left\{2^{n+1}\left(v_{n}-v_{n+1}\right)\right\}$ is a disjoint sequence of $L^{+}$and hence $\theta \leqq 2^{n+1}\left(v_{n}-v_{n+1}\right) \leqq u$ must hold in $L$ for some $u$. Also, $\theta \leqq\left(u_{n} \wedge w_{n}-\delta u_{1}\right)^{+} \leqq\left(u_{n}-\delta u_{1}\right)^{+} \wedge w_{n}=\theta$ implies $u_{n} \wedge w_{n} \leqq \delta u_{1}$ for all $n$. Thus,

$$
\begin{aligned}
\theta & \leqq u_{n}=u_{n} \wedge u_{1}=u_{n} \wedge\left(v_{n}+w_{n}\right) \leqq u_{n} \wedge v_{n}+u_{n} \wedge w_{n} \\
& \leqq v_{n}+\delta u_{1}=\left(v_{n}-v_{k}\right)+\delta u_{1}+v_{k}=\sum_{m=n}^{k-1}\left(v_{m}-v_{m+1}\right)+\delta u_{1}+v_{k} \\
& \leqq\left(\sum_{m=n}^{\infty} 2^{-m-1}\right) u+\delta u_{1}+v_{k}=2^{-n} u+\delta u_{1}+v_{k}
\end{aligned}
$$

holds in $L$ for all $k>n$. Now since $v_{n} \downarrow \theta$ we get $\theta \leqq u_{n} \leqq 2^{-n} u+\delta u_{1}$. Pick a positive integer $n_{1}$ with $2^{-n} u \in U$ for $n \geqq n_{1}$ and note that $\theta \leqq u_{n} \leqq 2^{-n} u+\delta u_{1} \in U+U \leqq V$ for $n \geqq n_{1}$. This implies $u_{n} \stackrel{\tau}{\rightarrow} \theta$ and the proof is finished.

REMARK. Note that the $\sigma$-Lebesgue property and pre-Lebesgue property together are not enough to imply the Lebesgue property [1, Example 2.5, p. 445]. This is, however, true for metrizable locally solid topologies [2, Theorem 6.6 , p. 121].

For the next result see also $[7$, p. 80$]$.

LEMMA 3.2. Let $(L, \tau)$ be a Hausdorff locally solid Riesz space with the Fatou property. Then we have:

(i) Every increasing $\tau$-bounded net of $L^{+}$is dominable.

(ii) If $L$ is $\sigma$-universally complete, then $\tau$ is a Lebesgue topology.

(iii) If $L$ is inextensible, then $(L, \tau)$ is $\tau$-complete. 
Proof. (i) Assume $\theta \leqq u_{\alpha} \uparrow$ is $\tau$-bounded and that $u>\theta$. Pick a Fatou $\tau$-neighborhood $V$ of zero with $u \notin V$ and a positive number $n$ with $n^{-1} u_{\alpha} \in V$ for all $\alpha$. Since $V$ satisfies the Fatou property there exists $\theta<w$ such that $n^{-1} u_{\alpha} \wedge u \leqq u-w$ for all $\alpha$. But then $\left(n u-u_{\alpha}\right)^{+}=n\left(u-u \wedge n^{-1} u_{\alpha}\right) \geqq n w>\theta$ holds for all $\alpha$, so that $\left\{u_{\alpha}\right\}$ is dominable.

(ii) Assume $u_{\alpha} \downarrow \theta$ in $L$ and that $V$ is a Fatou $\tau$-neighborhood of zero. By Theorem 3.1(i) $(L, \tau)$ satisfies the pre-Lebesgue property and so $\left\{u_{\alpha}\right\}$ is a $\tau$-Cauchy net. Thus $u_{\alpha}-u_{\beta} \in V$ for all $\alpha, \beta \geqq \alpha_{1}$. But then for each fixed $\alpha \geqq \alpha_{1}$ we have $u_{\tau}-u_{\beta} \underset{\beta}{\stackrel{(o)}{\longrightarrow} \alpha_{1}} u_{\alpha}$ holds in $L$. So for $\alpha \geqq \alpha_{1}$ we get $u_{\alpha} \in V$, i.e., $u_{\alpha} \stackrel{\tau}{\rightarrow} \theta$.

(iii) By [3, Theorem 4.1, p. 29] $L$ is order dense in its topological completion $\hat{L}$ and by Theorem 1.1(ii) $L=\hat{L}$, so that $(L, \tau)$ is $\tau$-complete.

LEMMA 3.3. Let $(L, \tau)$ be a $\sigma$-universally complete locally solid Riesz space and let $N=\bigcap\left\{V_{n}: n=1,2, \cdots\right\}$ be the null ideal of a normal sequence $\left\{V_{n}\right\}$ of solid $\tau$-neighborhoods of zero. Then $N^{d}$ is an Archimedean universally complete Riesz space with the countable sup property.

Proof. Observe that $N^{d}$ is a $\sigma$-universally complete Riesz space, since $N^{d}$ is a band. So it suffices to show that every disjoint subset of $N^{d}$ is at most countable. To this end let $A$ be a disjoint subset of $N^{d}$ of strictly positive elements. Note first that $A=\bigcup \mathbf{U}\left\{A_{n}: n=\right.$ $1,2, \cdots\}$, where $A_{n}=\left\{u \in A: u \notin V_{n}\right\}$, and then that each $A_{n}$ is finite (since every disjoint sequence converges to zero by Theorem 3.1). The universal completeness of $N^{d}$ is now immediate. For the countable sup property use [10, Theorem 29.3, 167] or [2, Theorem 6.6, p. 121].

We continue next with one of the main results of this section.

THEOREM 3.4. Let $(L, \tau)$ be a Hausdorff $\sigma$-universally complete locally solid Riesz space with the Fatou property. Assume that $\tau_{1}$ is another Hausdorff locally solid topology on $L$. Then $\tau_{1}$ is finer than $\tau$, i.e., $\tau \subseteq \tau_{1}$.

Proof. Assume $\theta \leqq u_{\alpha} \stackrel{\tau_{1}}{\rightarrow} \theta$ but $u_{\alpha} \stackrel{\tau}{\rightarrow} \theta$ does not hold. Then there exists a $\tau$-neighborhood $V$ of zero such that for any index $\alpha$ there exists $\beta \geqq \alpha$ with $u_{\beta} \notin V$. Now choose a normal sequence $\left\{V_{n}\right\}$ of Fatou $\tau$-neighborhoods of zero with $V_{1}+V_{1} \subseteq V$, let $N=\bigcap\left\{V_{n}: n=\right.$ $1,2, \cdots\}$ and note that by Lemma $3.3 N^{d}$ is a universally complete Riesz space with the countable sup property. Hence $N^{d}$ contains a weak order unit and is therefore a principal projection band [5, Theo- 
rem 1], i.e., $L=N \oplus N^{d}$. Put $u_{\alpha}=w_{\alpha}+v_{\alpha}, w_{\alpha} \in N ; v_{\alpha} \in N^{d}$. Observe that by Theorem 3.1(ii) $\tau_{1}$ induces a Lebesgue topology on $N^{d}$ (since $N^{d}$ has the countable sup property). Now by Theorem 2.2 there exists a metrizable Lebesgue topology $\tau^{\prime}$ on $N^{d}$ which is coarser than that induced by $\tau_{1}$, and so $v_{\alpha} \stackrel{\tau^{\prime}}{\rightarrow} \theta$. Pick a normal basis $\left\{U_{n}\right\}$ of zero for $\tau^{\prime}$ and then a sequence of indices $\left\{\alpha_{n}\right\}$ with $v_{\alpha_{n}} \in U_{n}$ and $u_{\alpha_{n}} \notin V$ for all $n$. Let $x_{n}=v_{\alpha_{1}}+\cdots+v_{\alpha_{n}}(n=1,2, \cdots)$ and note that $\theta \leqq x_{n} \uparrow$ and $\left\{x_{n}\right\}$ is $\tau^{\prime}$-bounded in $N^{d}$. By Lemma 3.2(i) $\left\{x_{n}\right\}$ is a dominable sequence of $N^{d}$ and by Theorem $1.2 \theta \leqq x_{n} \leqq x$ holds in $N^{d}$ for all

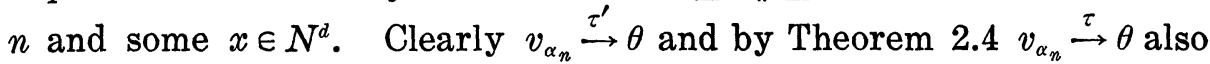
holds. But then $u_{\alpha_{n}} \in V$ for large $n$, in contradiction to the choice of $\left\{u_{\alpha_{n}}\right\}$. This contradiction establishes $\tau \leqq \tau_{1}$.

The next result follows immediately from the preceding theorem.

CoROLlary 3.5. A o-universally complete Riesz space admits at most one Hausdorff Fatou topology (which must be necessarily Lebesgue).

COROLLARY 3.6. For Hausdorff universally complete locally solid Riesz spaces $(L, \tau)$ the following statements are equivalent:

(i) $\tau$ is a metrizable topology.

(ii) $L$ has the countable sup property.

In particular a universally complete Riesz space with the countable sup property can admit at most one Hausdorff locally solid topology (which must be necessarily Lebesgue).

Proof. (i) $\Rightarrow$ (ii). Apply Lemma 3.3.

(ii) $\Rightarrow$ (i). From Theorem 3.1(ii) $(L, \tau)$ satisfies the Lebesgue property and so by Theorem $2.2 L$ admits a metrizable Lebesgue topology $\tau^{\prime}$. Corollary 3.5 now shows that $\tau=\tau^{\prime}$ and so $\tau$ is metrizable.

It is natural to ask when may a Hausdorff Fatou topology on a Riesz space $L$ be extended to a Hausdorff Fatou topology on its universal completion $L^{u}$. Evidently the topology on $L$ must be necessarily Lebesgue, and Corollary 3.5 shows that an Archimedean Riesz space can admit at most one such topology. The next theorem tells us what Hausdorff Lebesgue topology has such an extension.

THEOREM 3.7. Let $L$ be with a Hausdorff Lebesgue topology $\tau$ and with universal completion $L^{u}$. Then the following statements are equivalent:

(1) $\tau$ extends to a Lebesgue topology on $L^{u}$. 
(2) $\tau$ extends to a locally solid topology on $L^{u}$.

(3) Every dominable set of $L^{+}$is $\tau$-bounded.

(4) Every disjoint sequence of $L^{+} \tau$-converges to zero.

(5) Every disjoint sequence of $L^{+}$is $\tau$-bounded.

(6) The topological completion $\hat{L}$ of $L$ is Riesz isomorphic to $L^{u}$, i.e., $\hat{L}$ is the universal completion of $L$.

Proof. $\quad(1) \Rightarrow(2)$. Obvious.

$(2) \Rightarrow(3)$. By Theorem 1.2 every dominable set of $L^{+}$is order bounded in $L^{u}$ and hence bounded with respect to any locally solid topology on $L^{u}$.

$(3) \Rightarrow(4)$. If $\left\{u_{n}\right\}$ is a disjoint sequence of $L^{+}$, then so is $\left\{n u_{n}\right\}$. But since $\left\{n u_{n}\right\}$ is order bounded in $L^{u}$ it is dominable and hence by hypothesis $\tau$-bounded. Now let $V$ be a solid $\tau$-neighborhood of zero. Pick $k>0$ such that $n u_{n} \in k V$ for all $n$. But then $u_{n} \in V$ for $n \geqq k$ and thus $u_{n} \stackrel{\tau}{\rightarrow} \theta$.

$(4) \Rightarrow(5)$. Obvious.

$(5) \Rightarrow(6)$. Let $(\hat{L}, \hat{\tau})$ be the topological completion of $(L, \tau)$. Then $L$ is order dense in $\hat{L}$ [3, Theorem 4.1, p. 29], $(\hat{L}, \hat{\tau})$ satisfies the Lebesgue property and $\hat{L}$ is Dedekind complete [2, Theorems 3.1 and 3.2 , pp. 113-114]. So, it suffices to show that $\hat{L}$ is universally complete. To this end observe first that since $L$ is order and topologically dense in $\hat{L}$ every disjoint sequence of $\hat{L}^{+}$is $\hat{\tau}$-bounded. Now assume that $\left\{\hat{u}_{\alpha}: \alpha \in A\right\}$ is a disjoint system of $\hat{L}^{+}$. Let $\Delta$ be the set of all finite subsets of the indexed set $A$. For each $\delta \in \Delta$ put $\hat{v}_{\delta}=\sum_{\alpha \in \delta} \hat{u}_{\alpha}$. Then $\theta \leqq \hat{v}_{\hat{o}} \uparrow$ and we claim that $\left\{\hat{v}_{\hat{\delta}}: \delta \in \Delta\right\}$ is in fact a $\hat{\tau}$-Cauchy net. Indeed, if $\left\{\hat{v}_{\hat{o}}\right\}$ is not a $\hat{\tau}$-Cauchy net then there exists a solid $\tau$-neighborhood of zero $V$ such that for any $\delta \in \Delta$ there exist $\lambda, \mu \in \Delta$ with $\delta \subseteq \lambda, \delta \subseteq \mu$ and with $\hat{v}_{\lambda}-\hat{v}_{\mu} \notin \bar{V}(=$ the $\hat{\tau}$-closure of $V$ in $\hat{L})$. Thus there exists a sequence $\left\{\delta_{n}\right\}$ of $\Delta$ such that $\delta_{n} \subseteq \delta_{n+1}$ and with $\hat{w}_{n}=\hat{v}_{\delta_{n+1}}-\hat{v}_{\delta_{n}} \notin \bar{V}$ for all $n$. But $\left\{\hat{w}_{n}\right\}$ is a disjoint sequence of $\hat{L}^{+}$and hence so is $\left\{n \hat{w}_{n}\right\}$. Thus by what was noted above $\left\{n \hat{w}_{n}\right\}$ is $\hat{\tau}$-bounded and so $\left\{n \hat{w}_{n}\right\} \subseteq k \bar{V}$ holds for some $k>0$. Hence $\widehat{w}_{n} \in \bar{V}$ for large $n$, a contradiction. Hence $\left\{\widehat{v}_{\hat{\delta}}\right\}$ is a $\hat{\tau}$-Cauchy net and so $\hat{v}_{\delta} \stackrel{\hat{\tau}}{\rightarrow} \widehat{u}$ holds in $\hat{L}$. It follows now that $\sup \left\{\hat{u}_{\alpha}\right\}=\hat{u}$, so that $\hat{L}$ is universally complete. $(6) \Rightarrow(1)$. Observe that $\hat{\tau}$ is a Lebesgue extension of $\tau$ to $\hat{L}$.

Note. If a Hausdorff Lebesgue topology $\tau$ on a Riesz space $L$ extends to a Lebesgue topology $\tau^{u}$ on its universal completion $L^{u}$, then any basis $\{V\}$ of zero for $\tau$ consisting of Fatou neighborhoods extends to a basis $\left\{V^{u}\right\}$ of zero for $\tau^{u}$ consisting of Fatou neighborhoods of zero, where

$$
V^{u}=\left\{u^{*} \in L^{u}: \exists\left\{u_{\alpha}\right\} \subseteq V \quad \text { with } \quad \theta \leqq u_{\alpha} \uparrow\left|u^{*}\right| \text { in } L^{u}\right\} .
$$


THEOREM 3.8. If a $\sigma$-universally complete Riesz space L admits a Hausdorff Fatou topology $\tau$ then there exists an order dense $\sigma$-ideal of $L$ such that any other Hausdorff locally solid topology on $L$ induces the same topology (necessarily Lebesgue) on this ideal as $\tau$.

Proof. By Lemma 3.2(ii) $\tau$ is a Lebesgue topology and so the carrier $C_{\tau}$ of $\tau$ is an order dense $\sigma$-ideal of $L$ with the countable sup property (Lemma 2.1). In particular observe that $C_{-}$is a $\sigma$-universally complete Riesz space. If now $\tau_{1}$ is any other Hausdorff locally solid topology on $L$, then by Theorem 3.1(ii) $\left(L, \tau_{1}\right)$ satisfies the $\sigma$-Lebesgue property and hence $\tau_{1}$ restricted to $C_{-}$must be a Lebesgue topology. Now apply Corollary 3.5 to complete the proof.

We close this section with two open questions.

(1) Is every Hausdorff locally solid topology on an inextensible Riesz space necessarily Lebesgue? (See also [7, §3].)

(2) If an inextensible Riesz space admits a Hausdorff locally solid topology does it follow that it admits a Hausdorff Lebesgue topology also?

\section{REFERENCES}

1. C. D. Aliprantis, Some order and topological properties of locally solid linear topological Riesz spaces, Proc. Amer. Math. Soc., 40 (1973), 444-447.

2. - On the completion of Hausdorff locally solid Riesz spaces, Trans. Amer. Math. Soc., 196 (1974), 105-125.

3. C. D. Aliprantis and O. Burkinshaw, A new proof of Nakano's theorem in locally solid Riesz spaces, Math. Z., 144 (1975), 25-33.

4. I. Amemiya, On ordered topological linear spaces, In: Proceedings of the International Symposium on Linear Spaces (Jerusalem 1960) 14-23. Jerusalem: Israel Academy of Sciences and Humanities, 1961.

5. S. J. Bernau, Lateral and Dedekind completion of Archimedean lattice groups, J. London Math. Soc., 12 (1976), 320-322.

6. D. H. Fremlin, Topological Riesz Spaces and Measure Theory, Cambridge University Press, London, 1974.

7. _ Inextensible Riesz spaces, Math. Proc. Cambridge Philos. Soc., 77 (1975), 71-89.

8. A. M. Gleason, Projective topological spaces, Illinois J. Math., 2 (1958), 482-489.

9. W. A. J. Luxemburg and A. C. Zaanen, Notes on Banach function spaces, Nederl. Akad. Wetensch. Proc., Ser. A, Note X, 67 (1964), 493-506.

10. - Riesz Spaces I, North-Holland, Amsterdam, 1971,

11. A. I. Veksler and V. A. Geiler, Order and disjoint completeness of linear partially ordered groups, Siberian Math. J., 13 (1972), 30-35.

12. B. Z. Vulikh, Introduction to the theory of partially ordered spaces, Wolter-Noordhoff, Groningen, Netherlands, 1967. (English translation from the Russian.)

Received July 2, 1976 and in revised form January 17, 1977.

Indiana University-PuRdue UnIVERSity at INDIANAPOLIS

INDIANAPOLIS, IN 46205 


\section{PACIFIC JOURNAL OF MATHEMATICS}

\section{EDITORS}

RICHARD ARENS (Managing Editor)

University of California

Los Angeles, California 90024

C. W. CURTIS

University of Oregon

Eugene, OR 97403

C. C. MOORE

University of California

Berkeley, CA 94720

\section{J. DugundJI}

Department of Mathematics University of Southern Californıa Los Angeles, California 90007

R. FinN AND J. Milgram Stanford University Stanford, California 94305

\section{ASSOCIATE EDITORS}

E. F. BECKENBACH

B. H. NEUMANN

F. WOLF

K. YOSHIDA

\section{SUPPORTING INSTITUTIONS}

UNIVERSITY OF BRITISH COLUMBIA CALIFORNIA INSTITUTE OF TECHNOLOGY UNIVERSITY OF CALIFORNIA MONTANA STATE UNIVERSITY UNIVERSITY OF NEVADA, RENO NEW MEXICO STATE UNIVERSITY OREGON STATE UNIVERSITY UNIVERSITY OF OREGON OSAKA UNIVERSITY
UNIVERSITY OF SOUTHERN CALIFORNIA STANFORD UNIVERSITY UNIVERSITY OF TOKYO UNIVERSITY OF UTAH WASHINGTON STATE UNIVERSITY UNIVERSITY OF WASHINGTON AMERICAN MATHEMATICAL SOCIETY NAVAL WEAPONS CENTER 


\section{Pacific Journal of Mathematics}

\section{Vol. 71, No. $1 \quad$ November, 1977}

Charalambos D. Aliprantis and Owen Sidney Burkinshaw, On universally complete Riesz spaces ............................. 1

Stephen Richard Bernfeld and Jagdish Chandra, Minimal and maximal solutions of nonlinear boundary value problems .................

John H. E. Cohn, The length of the period of the simple continued fraction of

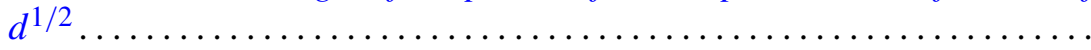

Earl Vern Dudley, Sidon sets associated with a closed subset of a compact abelian group .................................... 33

Larry Finkelstein, Finite groups with a standard component of type $J_{4} \ldots \ldots$

Louise Hay, Alfred Berry Manaster and Joseph Goeffrey Rosenstein, Concerning partial recursive similarity transformations of linearly ordered sets .......................................

Richard Michael Kane, On loop spaces without $p$ torsion. II ............

William A. Kirk and Rainald Schoneberg, Some results on

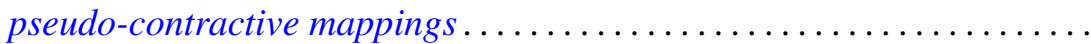

Philip A. Leonard and Kenneth S. Williams, The quadratic and quartic character of certain quadratic units. I. .

Lawrence Carlton Moore, A comparison of the relative uniform topology and the norm topology in a normed Riesz space .................

Mario Petrich, Maximal submonoids of the translational hull 119

Mark Bernard Ramras, Constructing new R-sequences . . .

Dave Riffelmacher, Multiplication alteration and related rigidity properties

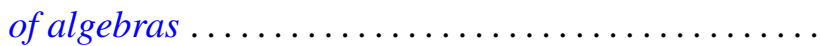

Jan Rosiński and Wojbor Woyczynski, Weakly orthogonally additive functionals, white noise integrals and linear Gaussian stochastic processes.

Ryōtarō Satō, Invariant measures for ergodic semigroups of operators

Peter John Slater and William Yslas Vélez, Permutations of the positive integers with restrictions on the sequence of differences...

Edith Twining Stevenson, Integral representations of algebraic cohomology classes on hypersurfaces ........................

Laif Swanson, Generators of factors of Bernoulli shifts . 